\title{
Protocol for a Cluster Randomized Trial Comparing Team-Based to Clinician-Focused Implementation of Advance Care Planning in Primary Care
}

\author{
Annette M. Totten, PhD, ${ }^{1}$ Lyle J. Fagnan, MD, ${ }^{2}$ David Dorr, MD, MS, ${ }^{3}$ LeAnn C. Michaels, BS, ${ }^{2}$ \\ Shigeko (Seiko) Izumi, PhD, RN, ${ }^{4}$ Angela Combe, MS, ${ }^{2}$ and France Légaré, MD, MSc, $\mathrm{PhD}^{5}$
}

\begin{abstract}
Introduction: For many patients, primary care is an appropriate setting for advance care planning (ACP). ACP focuses on what matters most to patients and ensuring health care supports patient-defined goals. ACP may involve interactions between a clinician and a patient, but for seriously ill patients ACP could be managed by a team.

Methods: We are conducting a cluster randomized trial comparing team-based to clinician-focused ACP using the Serious Illness Care Program (SICP) in 42 practices recruited from 7 practice-based research networks (PBRNs). Practices were randomized to one of the two models. Patients are referred to the study after engaging in ACP in primary care. Our target enrollment is 1260 subjects. Patient data are collected at enrollment, six months and one year. Primary outcomes are patient-reported goal-concordant care and days at home. Secondary outcomes include additional patient measures, clinician/team experience, and practice-level measures of SICP implementation.

Study Implementation: This trial was designed and is conducted by the Meta-network Learning and Research Center (Meta-LARC), a consortium of PBRNs focused on integrating engagement with patients, families, and other stakeholders into primary care research and practice. The trial pairs a comparative effectiveness study with implementation of a new program and is designed to balance fidelity to the assigned model with flexibility to allow each practice to adapt implementation to their environment and priorities. Our dissemination will report the results of comparing the two models and the implementation experience of the practices to create guidance for the spread of ACP in primary care.
\end{abstract}

Keywords: advance care planning; cluster randomized trial; Practice-Based Research Networks; primary care; serious illness care; team care

\section{Introduction}

$\mathbf{M}$ ANY PEOPLE have chronic conditions that are diagnosed months or years before death and have known, probable trajectories of decline. ${ }^{1}$ Often the default is to provide all available tests and treatments and health care risks harming patients, worsening quality of life, and increasing suffering. ${ }^{2}$ Furthermore, disregarding patients' preferences can be considered a medical error. ${ }^{3}$ Research has demonstrated that many patients and families want to discuss their goals and preferences $^{4}$ and that clinicians view such discussions as an important part of their practice. ${ }^{5}$ Nevertheless, assuring care honors what matters most to patients is not routine.

The mismatch between patient goals and health care is not inevitable. Health care can be a positive force even when life expectancy is limited as palliative care and related models of care have demonstrated. ${ }^{6-9}$ However, this requires advance care planning (ACP), a process to identify what is most important to patients and communicate this to family members and health care professionals. ACP is a necessary first step to

\footnotetext{
${ }^{1}$ Department of Medical Informatics and Clinical Epidemiology, Oregon Rural Practice-Based Research Network, Oregon Health and Science University, Portland, Oregon.

${ }^{2}$ Oregon Rural Practice-Based Research Network, Oregon Health and Science University, Portland, Oregon.

${ }^{3}$ Department of Medical Informatics and Clinical Epidemiology, Oregon Health and Science University, Portland, Oregon.

${ }^{4}$ School of Nursing, Oregon Health and Science University, Portland, Oregon.

${ }^{5}$ Centre de Recherche Sur Les Soins et Les Services de Première Ligne de l'Université Laval, Quebec, Canada.

Accepted June 17, 2019.
} 
provide care that is consistent with patient-defined goals and values. $^{10-13}$

Primary care is an appropriate setting for ACP as it is where most patients receive most of their care most of the time. ${ }^{14}$ Primary care clinicians have long-term trust-based relationships with their patients, understand the trajectory of illness, and want to have ACP conversations. ${ }^{5,15,16}$ However, they may not have the time, self-efficacy, skills, or confidence needed to engage patients in ACP. ${ }^{17-20}$

Care for patients with complex disease is increasingly provided by interprofessional teams. Yet ACP is traditionally viewed as limited to an interaction between a clinician and patient. Team-based ACP, tailored to primary care, may be able to increase ACP by distributing the time required and utilizing the skills of different professions. While some initial efforts have begun to explore how teams could be used for $\mathrm{ACP},{ }^{15,21}$ no large trials have evaluated teams conducting ACP in primary care.

This comparative effectiveness study is designed to address this gap by comparing two models for implementing an existing ACP program, the Serious Illness Care Program (SICP), developed by Ariadne Labs (www.ariadnelabs.org). The study is recruiting adults with serious illnesses receiving care from community-based primary care practices. The intervention is a team-based model of SICP and the comparator is a clinician-focused approach. Our hypothesis is that teambased SICP will be more effective, which could justify the changes and resources needed to implement a team-base model of ACP. Our primary patient outcomes are patientreported goal-concordant care and days at home.

\section{Methods \\ Study design}

We are conducting a cluster randomized trial (cRT) in which the clusters are primary care practices randomized to either a team-based or a clinician-focused model of SICP. This is a hybrid study in which we are studying both SICP implementation across practices and the comparative effectiveness of the two models. ${ }^{22,23}$ A cRT is appropriate as each model requires different training and changes in operations that impact the entire practice. Participating primary care practices were recruited from seven practice-based research networks (PBRNs) in five U.S. states (OR, WI, CO, IA, and NC) and two Canadian provinces (QB, ON). Study oversight, arm differentiation, enrollment, randomization, and data management are conducted by a Joint Coordinating Center (JCC) at Oregon Health \& Science University and Université Laval.

\section{Intervention and comparator}

The intervention and comparator are different models for implementation of the SICP. SICP is a comprehensive approach to ACP that requires (1) training and implementation support; (2) identifying appropriate patients; (3) using a structured conversation guide (the Serious Illness Conversation Guide); (4) creating workflows that allow serious illness conversations and follow-up; and (5) revisiting the conversation and revising any related decisions as patients' conditions and preferences change. SICP was developed by Ariadne Labs based on best practices, research on palliative care and patient/clinician communication, ${ }^{24-28}$ and data on the needs of patients, families, and clinicians. ${ }^{5,29,30}$ While SICP was initially designed and studied in oncology, it was developed to be used in various settings by clinicians who are not necessarily palliative care specialists.

The randomly assigned implementation model, either clinicianfocused or team-based, applies to all elements of SICP. This is demonstrated in Table 1, which outlines how the SICP elements differ across the arms.

The clinician-focused model centers serious illness care conversations between a primary care clinician (physicians, nurse practitioners, or physician assistants) and the patient and family. In this model, the primary care clinician identifies appropriate patients, has one or more conversation(s), and makes plans for future care and follow-up dicussions. This is the usual approach to ACP.

In the team-based model, primary care team members share SICP tasks appropriate to their scope of practice and coordinate communication. Teams consist of primary care clinicians and one or more additional persons from a different professions or practice roles (e.g., nurses, care managers, social workers, medical assistants, chaplains, peer counselors, community health workers, etc.). The team-based approach focuses on maximizing the expertise and efficient use of time of each team member to facilitate serious illness care planning. Although team-based care is becoming more common in primary care, particularly for multiple chronic illnesses, it is still not universal.

Implementation of both models involves a short initial training followed by practice facilitation (PF); these are specific to the assigned arm. Initial training was designed based on the SICP materials, with new videos and scenarios developed for the team-based arm. The initial training consists of a 1.5-hour online module and a 1.5-hour in-person session for role-play and discussion. The structure and content were designed to be feasible for busy primary care practices. PF is used to implement both models and track fidelity for this trial. PF involves practical support provided by trained practice facilitators as a way to bridge the gap between research and practice. ${ }^{31,32} \mathrm{PF}$ is a core component of $\mathrm{PBRNs}^{33}$ and all participating PBRNs have used PF before this trial. Practice facilitors serve as implementation agents, ${ }^{34}$ using a range of approaches and methods to build the internal capacity of practices for research, quality improvement, and practice transformation. This model is collaborative in that it forges alliances among practices in a geographic area and efficient as practice facilators are shared across practices. Table 1 outlines the focus of PF and fidelity tracking related to each SICP element.

\section{Outcomes}

The models are being implemented at the cluster level, but the primary outcomes are measured at the individual patient level and compared across the two models. The primary outcomes are: (1) goal-concordant care as reported by the patient (instrument developed for this trial), and (2) days spent at home in the prior six months. Days at home has been used as an outcome because most patients prefer to minimize time spent in the hospital or emergency department in the last years of life. ${ }^{35}$ Both will be measured six months and one year after enrollment. 
Table 1. Serious Illness Program Elements: Differences by Study Arm, Facilitation, and Fidelity Tracking

\begin{tabular}{|c|c|c|c|c|}
\hline SICP element & $\begin{array}{c}\text { Clinician-focused } \\
\text { arm }\end{array}$ & Team-based arm & Facilitation focus & Fidelity tracking \\
\hline Training & $\begin{array}{l}\text { PCCs } \\
\text { Part A (online, } \\
\text { video) } \\
\text { Part B (in person } \\
\quad \text { role play) }\end{array}$ & $\begin{array}{l}\text { All identified care team } \\
\text { members } \\
\text { Parts A (online, new } \\
\text { team video) } \\
\text { Part B (in person role } \\
\text { play with team roles) }\end{array}$ & $\begin{array}{l}\text { Identification of staff for } \\
\text { training, scheduling, } \\
\text { and. monitoring for } \\
\text { training needs (new } \\
\text { staff or refresher } \\
\text { training) }\end{array}$ & $\begin{array}{l}\text { Arm-specific training } \\
\text { completed by } \\
\text { appropriate staff }\end{array}$ \\
\hline $\begin{array}{l}\text { Patient } \\
\text { identification }\end{array}$ & $\begin{array}{l}\text { Individual PCCs } \\
\text { responsible for } \\
\text { identification of } \\
\text { eligible patients }\end{array}$ & $\begin{array}{l}\text { Team members } \\
\text { contribute to } \\
\text { identification of } \\
\text { eligible patients. }\end{array}$ & $\begin{array}{l}\text { Feasible process for } \\
\text { identifying eligible } \\
\text { patients using available } \\
\text { resources: clinical } \\
\text { intuition, patient lists, } \\
\text { or algorithms }\end{array}$ & $\begin{array}{l}\text { Documentation } \\
\text { of patient } \\
\text { identification } \\
\text { process and any } \\
\text { modifications. } \\
\text { Focus on arm- } \\
\text { specific roles }\end{array}$ \\
\hline $\begin{array}{l}\text { Patient invitation/ } \\
\text { pre-conversation } \\
\text { preparation }\end{array}$ & Introduction by $\mathrm{PCC}$ & $\begin{array}{l}\text { Introduction by any team } \\
\text { member }\end{array}$ & $\begin{array}{l}\text { Development of } \\
\text { workflows and } \\
\text { invitation modes } \\
\text { (letter, call, visits) }\end{array}$ & $\begin{array}{l}\text { Documentation } \\
\text { of arm-appropriate } \\
\text { workflow and } \\
\text { modifications }\end{array}$ \\
\hline Patient interaction & $\begin{array}{l}\text { PCC has initial } \\
\text { Serious Illness, } \\
\text { Conversation(s) } \\
\text { and plans follow- } \\
\text { up. Other staff } \\
\text { minimally } \\
\text { involved (e.g., } \\
\text { scheduling) }\end{array}$ & $\begin{array}{l}\text { Practices plan and } \\
\text { standardize } \\
\text { conversations and } \\
\text { follow-up to be } \\
\text { purposefully shared } \\
\text { across team members. }\end{array}$ & $\begin{array}{l}\text { Development } \\
\text { of arm-appropriate } \\
\text { workflows and } \\
\text { variations }\end{array}$ & $\begin{array}{l}\text { Documentation } \\
\text { of actual workflows } \\
\text { and patient } \\
\text { trajectories during } \\
\text { study period. Focus } \\
\text { on adherence to } \\
\text { assigned model }\end{array}$ \\
\hline $\begin{array}{l}\text { Communication of } \\
\text { preferences }\end{array}$ & $\begin{array}{l}\text { External: PCC } \\
\text { determines how to } \\
\text { communicate } \\
\text { patient preferences } \\
\text { to other settings } \\
\text { and specialists }\end{array}$ & $\begin{array}{l}\text { External: Team } \\
\text { communication } \\
\text { processes to other } \\
\text { settings and specialists } \\
\text { Internal: Structured } \\
\text { team communication/ } \\
\text { coordination. }\end{array}$ & $\begin{array}{l}\text { Development } \\
\text { of arm-appropriate } \\
\text { communication } \\
\text { strategies and } \\
\text { variations }\end{array}$ & $\begin{array}{l}\text { Documentation } \\
\text { of actual } \\
\text { communications } \\
\text { patterns }\end{array}$ \\
\hline Documentation & $\begin{array}{l}\text { Sufficient to } \\
\text { facilitate PCC } \\
\text { follow-up, } \\
\text { generate } \\
\text { reminders, bill }\end{array}$ & $\begin{array}{l}\text { Sufficient to facilitate } \\
\text { team follow-up, } \\
\text { generate reminders, } \\
\text { bill }\end{array}$ & $\begin{array}{l}\text { Development } \\
\text { of arm-appropriate } \\
\text { workflows and } \\
\text { acceptable variations }\end{array}$ & $\begin{array}{l}\text { Documentation } \\
\text { of actual workflows } \\
\text { and patient } \\
\text { trajectories during } \\
\text { study period }\end{array}$ \\
\hline
\end{tabular}

PCC, primary care clinician; SICP, Serious Illness Care Program.

Secondary outcomes include additional patient, clinician/ team member, and practice-level outcomes. Secondary patient outcomes include anxiety, depression, and self-reported health (PROMIS-29), ${ }^{36-38}$ use of health care services (including hospice, palliative care, and primary care), preferences for medical decision making (decision control preferences scale), ${ }^{39}$ and patient experience with SICP. In addition, all identified care partners will be asked about their experience as a caregiver (Zarit Burden Interview scale), ${ }^{40-42}$ their own health (PROMIS-29), and their own experience with SICP. If we learn of a patient death, either from the primary care practice or in the course of follow-up attempts, care partners identified by participating patients will be asked to report hospice use, location of death, and family bereavement. Clinician and team member outcomes will include intention to engage patients and use SICP, their perceptions of SICP effectiveness, and reported use of SICP. Practice-level outcomes are measures that SICP has successfully implemented and includes the achievement of the key components of the program: the ability to identify appropriate patients, to engage patients and families in serious illness conversations, to document patient goals and preferences in medical records, and to create workflows that facilitate these activities as well as appropriate follow-up.

\section{Recruitment and Study Procedures \\ Primary care practice eligibility and recruitment}

To be eligible for inclusion, primary care practices needed: sufficient number of patients likely to meet eligibility requirements; a willingness to be randomized to either arm (e.g., ability to create a clinical ACP team); and not be currently engaged in a standardized ACP program. Participating practices could be in the same health system, but could not share staff or physical space. Interested practices were identified and assessed by the PBRN director and an application outlining practice interest and characteristics was submitted for approval by the study JCC. Six practices from each of 7 PBRN (total 42 practices) were recruited during the first year of the study.

Operations Groups at each PBRN (including at minimum a director, practice facilitator, coordinator, and patient/family 
representative) oversee the implementation of SICP according to the assigned arm and monitor study procedures, including subject referral and data collection. Members of the Operations Groups participate in the quarterly trial steering committee meetings and monthly PBRN leadership meetings. This infrastructure is needed for the trial and will not be required to spread the model to other practices once the trial is complete.

Practices accepted for participation were randomly assigned to either the team-based or clinician-focused arm by a blinded biostatistician who had no contact with the practices or PBRNs. Allocation was 1:1 for clinician- and team-based arms and was stratified by PBRN. This statistician was provided with practice ID numbers and baseline practice characteristics, then used computer-generated random numbers to assign practices into two groups without knowing which group will be assigned to which model. Investigators, PBRN leadership, practices, and research staff cannot be blinded to assignment as they are implementing the assigned SICP model. Patients are told they are participating in a study designed to improve ACP, but they are not explicitly told that the study will compare two models.

Once enrolled, each practice creates a small practice study group consisting of at least one administrator, one clinical staff member (nurse and/or medical assistant), and one clinician. This group works with practice faciliators to design workflows for SICP implementation that corresponds to the assigned arm and the environment of each practice. This strucuture is designed to be similar to what would be required to implement any new program in the practice and members of this group do not necessarily participate in SICP, although they may if appropriate. Practices are compensated for data collection activities that fall outside of normal care delivery.

\section{Patient eligibility and recruitment}

At enrollment, patients must be adults (over 18 years old), community dwelling (e.g., not residing in a nursing home), and not in hospice, or in a hospital with no plans for discharge. Patients may have any serious illnesses or conditions that are likely to limit their life expectancy to less than two years as defined by using clinical intuition (e.g., they would not be surprised if the patient died in the next two years $)^{43}$ alone, or supplemented by an available algorithm. ${ }^{4,45} \mathrm{~Pa}-$ tients who have ACP documents or end-of-life care-related medical orders (e.g., advance directives, do not resuscitate order or Portable Orders for Life Sustaining Treatment) are not excluded as options and values may change over time. Family members or friends involved in care, referred to as care partners, can be recruited only if a patient agrees that this person can be contacted for the study.

Practices may engage as many patients and families in SICP as they wish and all subsequent care is expected to be provided at the practice's discretion. After patients have serious illness conversation with their clinician or team, clinicians or other practice staff briefly introduce the study and, if the patient is interested, obtain permission to share contact information with research staff. Practices securely transmit patient contact information to the PBRN staff using means that comply with human subject protections. PBRN researchers contact patients to describe the study, obtain consent, and collect initial information and baseline survey data.
Patient enrollment methods differ between the U.S. and Canada: in the U.S. the researchers make the initial contact by phone and collect verbal consent while in Canada the researchers schedule an in-person appointment for written consent and enrollment. In both countries, patients will be contacted six months and one year after enrollment by the PBRN for additional surveys. The ongoing care and clinical follow-ups needed by the patient are determined by the practice and are part of the study intervention only to the extent that they are informed by ACP achieved using SICP. Patients will only be removed from the trial if they request no further contact with the study.

\section{Data Collection and Analysis}

Data will be collected from patients, care partners named by patients, care partners of deceased patients, and clinicians and teams at the primary care practices. Patients and care partners will complete questionnaires at enrollment and again at six months and one year after enrollment. These may be done by phone, electronically, or mail. If a patient dies during the study period, we will attempt to conduct a bereavement interview with a care partner within 6 to 12 weeks after the patient's death. Clinicians and team members will be surveyed after training and one and two years later. Data about participating practices (e.g., size, ownership, electronic health record (EHR), clinic care team composition, patient demographics) will be collected at recruitment. Information about any disruptions at the practices likely to impact SICP implementation (e.g., change in leadership, ownership, or EHR) will be collected during quarterly visits. Patients will be recruited during a 12-month period and data collection activities will continue for 18 months after the last patient is enrolled to allow for patient follow-up and surveys of the primary care staff. Besides the primary outcomes, any harms will be collected from the practices and the patients and reported to appropriate regulatory authorities as needed. Regular auditing will occur by a committee of study staff and stakeholders to assure that data integrity remains intact and problems are addressed.

Data are stored in two REDCap research databases, one maintained in the United States and one in Canada to allow tracking and monitoring of enrollment and follow-up. The JCC will use several methods to monitor data for quality and completeness, including comparing data to source documents, data range checks, and timeliness of data collection. At the completion of data collection, patient identifiers will be removed and the two databases will be merged for analysis.

\section{Sample size}

We estimated sample sizes to detect meaningful differences in two primary outcome measures, goal-concordant care, and days at home, specifying $\alpha=0.025$ for each. We determined that a $15 \%$-point difference in proportion of patients who report high goal concordance will be achievable and relevant. Assuming 50\% and 65\% for the two arms and an intracluster (practice) correlation coefficient (ICC) of 0.025 , we will be powered at $\geq 90 \%$ to detect this difference using a two-sided test with 34 practices having 23 patients each $(n=782)$. We approximated the model for our other primary outcome, days at home, as a proportion of time. Prior research reported rates for days at home for the last six months of life ranging from 118.8 to 145.9 in the United States. ${ }^{35}$ With 
an ICC of 0.01 that is typical of clinical outcomes, the same sample size gives $\geq 90 \%$ power to detect a difference from $80 \%$ of days at home (146 days/ 6 months) of $+10 \%$, or 14 additional days at home over 6 months. To achieve this sample size for analysis after potential attrition of clinics and patients, we randomized 42 practices equally to each arm and aim to enroll 30 patients at each practice $(n=1260)$.

\section{Analysis}

We will perform a descriptive analysis of practice, clinician, and patient characteristics to assure comparability of the two study arms and include those identified as important by stakeholders as covariates in analyses. Multilevel modeling will be used to account for the hierarchical structure of the data. We will specify random effects at the practice (cluster) level and, when comparing baseline to follow-up, at the respondent level. For each outcome, we will assess goodness of fit and model assumptions.

Primary analyses will be conducted under an intentionto-treat assumption, and thus missing data will be multiply imputed using baseline measurements and standard tools. Because missingness may be informative in this study, we also plan to evaluate potential bias with (1) a worst-case sensitivity analysis, in which the worst outcome is substituted for missing outcomes, and (2) a Heckman selection model, ${ }^{46}$ assessing the probability of having non-missing outcome data using available information, then test the correlation between the residuals of that model and those in our main outcome model. This approach will also allow us to compare biascorrected with uncorrected estimates of treatment effects.

We plan one interim analysis to determine whether to stop after we have collected six-month outcomes on 408 participants (12 per cluster). Specifically, we will perform a onesided test at $\alpha=0.05$ against a null hypothesis that the proportion of patients in both arms combined reporting goalconcordant care is 0.15 . Because we will not compare the two approaches, we do not need to unblind the statisticians or adjust significance levels of primary analyses. Failure to reject the null would mean that (1) neither arm is performing meaningful ACP, or (2) one arm is outperforming the other dramatically. While not expected, either case provides justification for stopping.

We will follow the CONSORT extension for $\mathrm{cRTs}^{47}$ when reporting the trial results.

\section{Ethics Determination and Trial Registration}

The study has been reviewed and approved by the Trial Innovation Network Single IRB at Vanderbilt University Medical Center (IRB\#181084) for the U.S. sites; by the Research Ethics Board of the Centre intégré universitaire de santé et de services sociaux (CIUSSS) de la Capitale-Nationale in Quebec City, Canada (ethics number \#MP-13-2019-1526) for the sites in Quebec; and by the Health Sciences Research Ethics Board of the University of Toronto (protocol number 36631) for the sites in Ontario. The trial is registered at ClinicalTrials.gov (ID: NCT03577002; date posted July 4, 2018)

\section{Trial Challenges and Innovations}

In addition to the methods outlined above, we will report on, key innovative elements of this trial designed to address the challenges of conducting research in diverse practices and sustaining practice change in primary care. These include our reliance on a consortium of PBRNs, engagement, fidelity assessment, and plans for dissemination.

\section{Building complex research using a consortium of PBRNs}

This trial is dependent on the infrastructure and relationships created by Meta-network Learning and Research Center (Meta-LARC), a consortium of seven PBRNs that strive to increase the quality, effectiveness, and safety of primary care through collaborative learning. Meta-LARC was founded in 2012 with support by a five-year award from the Agency for Health Care Research and Quality (AHRQ).

Meta-LARC facilitated the development of the proposal for this trial and is key to its execution. Operating through the PBRNs, we were able to assess the interest in ACP across primary care practices, identify key barriers to ACP, and explore potential interventions during proposal development. The trust-based relationships forged between the PBRNs and their 925 member practices have made diverse practice recruitment feasible; something that would be difficult if we had to recruit practices without prior contact or were limited to the practices affiliated with one institution or investigator.

Equally important, Meta-LARC and the history of collaboration among the PBRNs allows the coproduction of the comparative effectiveness trial and SICP implementation, which is key to practice engagement and sustainability. Leadership, coordinators and practice facilitators from the PBRNs meet to share experience, expertise, and troubleshoot problems. Key to the trial execution is that the PBRNs are the primary contact with the participating practices: providing training, supporting implementation of SICP adapted to each practice's context, and completing the research enrollment and data collection tasks. This allows "on the ground" involvement with a large number of practices during the trial, helps the PBRNs deepen their connections to their member practices, and contributes to the ongoing development of the capacity of the consortium and individual practices for more community-based research.

\section{Engagement}

Our approach reflects the complexity of achieving meaningful engagement in a research project and focuses on authentic integration of patients and families, as well as other stakeholders, into the study. Effectively incorporating engagement into a trial requires specialized skills, just as conducting complex analyses requires biostatistical knowledge. We included consultants from the Institute for Patient- and Family-Centered Care (www.ipfcc.org) as advisors and coaches to further develop engagement capacity, supplementing the engagement experience within the participating PBRN leadership. ${ }^{48-51}$

Engagement activities were developed in response to PCORI's charge and awareness that the success of a complex project requires engagement with patients, families/care partners, primary care practices, and external policy makers. We outlined an approach to engagement in our proposal, developed this into a detailed engagement plan after the project was funded, and are reviewing and updating the plan annually. 
Table 2. Engagement Goals and Key Activities

\begin{tabular}{lc}
\hline Goal for engagement & Key activities \\
\hline Develop SICP adaptation & Involve PFAs and other partners in development of \\
& The training materials \\
- Patient facing materials, for SICP and for study recruitment & ACP workflows \\
Focus on important outcomes that matter & Use numerous mechanisms (e.g., focus groups, surveys, and break-out sessions \\
to patients and their families & at meetings) to capture ideas, thoughts, suggestions from early stages \\
& through decision making on outcomes measurement, analysis, and reporting. \\
Assure successful implementation & Include PFAs in PBRN Operations Groups to ensure that their ideas are heard \\
of SICP & and integrated into workflows that address both patient needs and the \\
& differences across practices. \\
& Ensure clinicians and administrators provide critical perspectives so that SICP \\
implementation is feasible. & Support clinics in engaging their patients and their communities in promoting \\
ACP and the study. & Enhance study recruitment and retention strategies. \\
& Develop strong relationships with patient, family, clinician, and primary care \\
practice partners to increase comfort with SICP and ACP conversations. & Document implementation barriers and facilitators from multiple perspectives \\
to encourage replication and spread. & Explore ways of engaging patients, families, and PCCs and practices in this \\
conversation outside study parameters (ongoing communication). &
\end{tabular}

ACP, advance care planning; PBRN, practice-based research network; PFA, Patient and Family Advisor.

We operationalized engagement in terms of four goals and corresponding key activities outlined in Table 2. Engagement of patients and families centers on the inclusion of Patient and Family Advisors (PFAs) in the project steering committee and the seven PBRN Operations Groups. We aim to create flexible and transparent processes that ensure PFAs make meaningful contributions that benefit the conduct of the trial. ${ }^{52} \mathrm{We}$ are supporting involvement of patients at the participating practices through outreach to existing Patient and Family Advisory Councils (PFACs). If practices do not have PFACs, we offer help either developing a PFAC or identifying other mechanisms for engagement. After the initial planning and site recruitment, we are convening external stakeholders (e.g., national patient and family groups and policy makers) to advise on how documentation and dissemination can maximize the impact of the trial.

\section{Monitoring fidelity while allowing adaptation by practice}

A characteristic of this trial is the effort we made to balance standardization and flexibility across the participating practices. Certain elements need to be standardized to assure that fidelity is maintained to the SICP and to the randomization. Yet each practice needs the flexibility to adapt SICP to their own environment and needs.

Key to monitoring fidelity and documenting variation will be the monthly check-ins and quarterly meetings PBRNs will have with each participating practice. Information from these encounters will be collected and compiled in the study database to allow for ongoing review during the trial as well as analysis on completion. Table 1 includes an overview of facilitation and fidelity monitoring activites for each SICP element. The structured data collection will be reviewed and revised as needed over the course of the trial. Fidelity to the assigned model will be anchored to a detailed description developed following the TIDieR checklist. ${ }^{53}$ Variations in implementation will be analyzed and described using the PARIHS implementation framework ${ }^{54}$ and multimethod approaches to the assessment of adaptations. ${ }^{55}$ Variations from core components will be treated as protocol violations and monitored by a committee of stakeholders and reported to regulatory authorities.

\section{Plans for dissemination and spread}

We will publish and present the results of our trial in journals and at meetings that focus on primary care and on $\mathrm{ACP}$ and distribute the results to the participating practices and patients. Before the trial is completed, we will use articles and presentations to share several unique aspects of our study, including the development of our team training for SICP, our experience working with practices in the United States and Canada, and our efforts to develop a patientreported measure of goal-concordant care. We plan to use a two-pronged approach to increasing the spread of ACP in primary care. First, we will assist any participating practices that wish to share their efforts and experiences with their communities through local presentations and media. Our intention is to spotlight the practice involvement in research and the importance of ACP. Second, during the trial we will compile the characteristics and experiences of all the participating practices with a focus on the barriers and facilitators to implementation. This can serve as an implementation manual and provide a range of prototypes for primary care practices interested in ACP generally or SICP specifically.

\section{Acknowledgments}

The authors would like to acknowledge the contributions made by the consortium of Practice-Based Research 
Networks (PBRNs), the Meta-Network Learning and Research Center (Meta-LARC). Meta-LARC includes the following PBRNs:

Duke University, Duke Primary Care Research Consortium (PCRC); Oregon Health \& Science University, Oregon Rural Practice-based Research Network (ORPRN); University of Colorado, State Networks of Colorado Ambulatory Practices \& Partners (SNOCAP); University of Iowa, Iowa Research Network (IRENE); University of Laval, Québec Practice Based Research Network (QPBRN); University of Wisconsin, Wisconsin Research and Education Network (WREN); University of Toronto, University of Toronto Practice-Based Research Network (UTOPIAN). Research reported in this article was funded through a Patient-Centered Outcomes Research Institute ${ }^{\circledR}$ (PCORI ${ }^{\circledR}$ ) PCORI Award (PLC-1609-36277). The statements made in this protocol are solely the responsibility of the authors and do not necessarily represent the views of the Patient-Centered Outcomes Research Institute (PCORI), its Board of Governors or Methodology Committee or other participants in PCORnet ${ }^{\circledR}$. The use of REDCap in this trial was supported by National Center for Advancing Translational Sciences of the National Institutes of Health under award number UL1TR0002369.

Leadership, staff and member practices contributed to the design of this study and are now participating in the trial. Without their insight and contributions this project would not be possible. Additionally we would like to acknowledge the Institute for Patient- and Family-Centered Care (Mary Minniti and Deborah Dokken) for their advise and counsel on meaningful engagement.

\section{Author Disclosure Statement}

No competing financial interests exist

\section{References}

1. Heron M: Deaths: Leading causes for 2014. Natl Vital Stat Rep 2016;65:1-96.

2. Committee on Approaching Death: Addressing Key End of Life Issues: Dying in America: Improving Quality and Honoring Individual Preferences Near the End of Life. Washington, DC: Institute of Medicine, 2015.

3. Allison TA, Sudore RL: Disregard of patients' preferences is a medical error: Comment on "Failure to engage hospitalized elderly patients and their families in advance care planning”. JAMA Intern Med 2013;173:787.

4. PerryUndem Research/Communication: Conversation Staters: Research Insights from Clinicians and Patients on Converstations About End-of-Life Care and Wishes, Volume November. John A. Hartford Foundation, Californial Health Care Foundation, Cambia Health Foundation, 2016.

5. PerryUndem Research/Communication: Physicians' Views Toward Advance Care Planning and End-of-Life Care Conversations: Finding form a National Survey among Physicians Who Regularly Treat Patients 65 and Older, Volume April. John A. Hartford Foundation, Californial Health Care Foundation, Cambia Health Foundation, 2016.

6. Gomes B, Calanzani N, Curiale V, et al.: Effectiveness and cost-effectiveness of home palliative care services for adults with advanced illness and their caregivers. Cochrane Database Syst Rev 2013:CD007760.
7. McCarthy D, Ryan J, Klein S: Models of care for highneed, high-cost patients: An evidence synthesis. Issue Brief (Commonw Fund) 2015;31:1-19.

8. Meier DE, Back AL, Berman A, et al.: A national strategy For palliative care. Health Aff (Millwood) 2017;36:12651273.

9. Selvaggi KJ, Vick JB, Jessell SA, et al.: Bridging the gap: A palliative care consultation service in a hematological malignancy-bone marrow transplant unit. J Community Support Oncol 2014;12:50-55.

10. Billings JA, Bernacki R: Strategic targeting of advance care planning interventions: The Goldilocks phenomenon. JAMA Intern Med 2014;174:620-624.

11. Brinkman-Stoppelenburg A, Rietjens JA, van der Heide A: The effects of advance care planning on end-of-life care: A systematic review. Palliat Med 2014;28:1000-1025.

12. Frank RK: Shared decision making and its role in end of life care. Br J Nurs 2009;18:612-618.

13. Fromme EK, Zive D, Schmidt TA, et al.: Association between physician orders for life-sustaining treatment for scope of treatment and in-hospital death in Oregon. J Am Geriatr Soc 2014;62:1246-1251.

14. Green LA, Fryer GE, Jr., Yawn BP, et al.: The ecology of medical care revisited. N Engl J Med 2001;344:2021-2025.

15. Lakin JR, Benotti E, Paladino J, et al.: Interprofessional work in serious illness communication in primary care: A qualitative study. J Palliat Med 2019;22:751-763.

16. Sandoval MB, King JG, Hart V, et al.: Increasing advance care planning in primary care practices: A multi-site quality improvement initiative. J Gen Intern Med 2019;34:809-811.

17. Coleman K, Mattke S, Perrault PJ, et al.: Untangling practice redesign from disease management: How do we best care for the chronically ill? Annu Rev Public Health 2009;30:385-408.

18. Glasgow RE, Davis CL, Funnell MM, et al.: Implementing practical interventions to support chronic illness selfmanagement. Jt Comm J Qual Saf 2003;29:563-574.

19. Lee L, Hillier LM, Locklin J, et al.: Advanced care planning for persons with dementia in primary care: Attitudes and barriers among health-care professionals. J Palliat Care 2018:825859718812463.

20. Ostbye T, Yarnall KS, Krause KM, et al.: Is there time for management of patients with chronic diseases in primary care? Ann Fam Med 2005;3:209-214.

21. Dixon J, Knapp M: Whose job? The staffing of advance care planning support in twelve international healthcare organizations: A qualitative interview study. BMC Palliat Care 2018;17:78.

22. Curran GM, Bauer M, Mittman B, et al.: Effectivenessimplementation hybrid designs: Combining elements of clinical effectiveness and implementation research to enhance public health impact. Med Care 2012;50:217-226.

23. Loudon K, Treweek S, Sullivan F, et al.: The PRECIS-2 tool: Designing trials that are fit for purpose. BMJ 2015; 350:h2147.

24. Bernacki RE, Block SD, American College of Physicians High Value Care Task F: Communication about serious illness care goals: A review and synthesis of best practices. JAMA Intern Med 2014;174:1994-2003.

25. Austin CA, Mohottige D, Sudore RL, et al:: Tools to promote shared decision making in serious illness: A systematic review. JAMA Intern Med 2015;175:1213-1221.

26. Chung HO, Oczkowski SJ, Hanvey L, et al.: Educational interventions to train healthcare professionals in end-of-life 
communication: A systematic review and meta-analysis. BMC Med Educ 2016;16:131.

27. Fawole OA, Dy SM, Wilson RF, et al.: A systematic review of communication quality improvement interventions for patients with advanced and serious illness. J Gen Intern Med 2013;28:570-577.

28. Legare F, Stacey D, Turcotte S, et al.: Interventions for improving the adoption of shared decision making by healthcare professionals. Cochrane Database Syst Rev 2014:CD006732.

29. Sanders JJ, Robinson MT, Block SD: Factors impacting advance care planning among African Americans: Results of a systematic integrated review. J Palliat Med 2016;19: 202-227.

30. Schonberg MA, Smith AK: Discussing long-term prognosis in primary care: Hard but necessary. JAMA Intern Med 2016;176:678-680.

31. Nagykaldi Z, Mold JW, Aspy CB: Practice facilitators: A review of the literature. Fam Med 2005;37:581-588.

32. Baskerville NB, Liddy C, Hogg W: Systematic review and meta-analysis of practice facilitation within primary care settings. Ann Fam Med 2012;10:63-74.

33. Nagykaldi Z, Mold JW, Robinson A, et al.: Practice facilitators and practice-based research networks. J Am Board Fam Med 2006;19:506-510.

34. Proctor E, Silmere H, Raghavan R, et al.: Outcomes for implementation research: Conceptual distinctions, measurement challenges, and research agenda. Adm Policy Ment Health 2011;38:65-76.

35. Groff AC, Colla $\mathrm{CH}$, Lee TH: Days spent at home-A patient-centered goal and outcome. N Engl J Med 2016; 375:1610-1612.

36. Craig BM, Reeve BB, Brown PM, et al.: US valuation of health outcomes measured using the PROMIS-29. Value Health 2014;17:846-853.

37. Rose AJ, Bayliss E, Baseman L, et al.: Feasibility of distinguishing performance among provider groups using patient-reported outcome measures in older adults with multiple chronic conditions. Med Care 2019;57:180-186.

38. Rose AJ, Bayliss E, Huang W, et al.: Evaluating the PROMIS-29 v2.0 for use among older adults with multiple chronic conditions. Qual Life Res 2018;27:29352944.

39. Chiu C, Feuz MA, McMahan RD, et al.: "Doctor, Make My Decisions": Decision control preferences, advance care planning, and satisfaction with communication among diverse older adults. J Pain Symptom Manage 2016;51:3340.

40. Bedard M, Molloy DW, Squire L, et al.: The Zarit Burden Interview: A new short version and screening version. Gerontologist 2001;41:652-657.

41. O'Rourke N, Tuokko HA: Psychometric properties of an abridged version of The Zarit Burden Interview within a representative Canadian caregiver sample. Gerontologist 2003;43:121-127.
42. Zarit SH, Reever KE, Bach-Peterson J: Relatives of the impaired elderly: Correlates of feelings of burden. Gerontologist 1980;20:649-655.

43. Vick JB, Pertsch N, Hutchings M, et al.: The utility of the surprise question in identifying patients most at risk of death. J Clin Oncol 2015;33:abstr8.

44. Schonberg MA, Davis RB, McCarthy EP, et al.: External validation of an index to predict up to 9-year mortality of community-dwelling adults aged 65 and older. J Am Geriatr Soc 2011;59:1444-1451.

45. Lee SJ, Lindquist K, Segal MR, et al.: Development and validation of a prognostic index for 4-year mortality in older adults. JAMA 2006;295:801-808.

46. Heckman J: Sample selection bias as a specification error. Econometrica 1979;47:153-161.

47. Campbell MK, Piaggio G, Elbourne DR, et al.: Consort 2010 statement: Extension to cluster randomised trials. BMJ 2012;345:e5661.

48. Fagnan L, Simpson M, Daly J, et al.: Adapting boot camp translation methods to engage clinician/patient research teams within Practice-based research networks. J Patient Cent Res Rev 2018;5:209-303.

49. Haesebaert J, Samson I, Lee-Gosselin H, et al.: How to engage patients in research and quality improvement in community-based primary care settings: Protocol for a participatory action research pilot study. Res Involv Engagem 2018;4:30.

50. Hahn DL, Hoffmann AE, Felzien M, et al.: Tokenism in patient engagement. Fam Pract 2017;34:290-295.

51. Nease DE, Jr., Burton D, Cutrona SL, et al.: "Our lab is the community": Defining essential supporting infrastructure in engagement research. J Clin Transl Sci 2018;2:228-233.

52. Goodman MS, Sanders Thompson VL, Johnson CA, et al.: Evaluating community engagement in research: Quantitative measure development. J Community Psychol 2017;45: 17-32.

53. Hoffmann TC, Glasziou PP, Boutron I, et al.: Better reporting of interventions: Template for intervention description and replication (TIDieR) checklist and guide. BMJ 2014;348:g1687.

54. Harvey G, Kitson A: PARIHS revisited: From heuristic to integrated framework for the successful implementation of knowledge into practice. Implement Sci 2016;11:33.

55. Rabin BA, McCreight M, Battaglia C, et al.: Systematic, multimethod assessment of adaptations across four diverse health systems interventions. Front Public Health 2018;6: 102 .

Address correspondence to: Annette M. Totten, PhD

Oregon Health and Science University Department of Medical Informatics and Clinical Epidemiology

Oregon Rural Practice-based Research Network Portland, OR 97239-3098

E-mail: totten@ohsu.edu 